\title{
The Brief Dimensional Apathy Scale (b-DAS): a short clinical assessment of apathy
}

Ratko Radakovic ${ }^{1,2,3,5^{*}}$, Sarah McGrory ${ }^{4}$, Siddharthan Chandran ${ }^{3}$, Robert Swingler ${ }^{3}$, Suvankar Pal ${ }^{3}$, Laura Stephenson ${ }^{3}$, Shuna Colville ${ }^{3}$, Judy Newton ${ }^{3}$, John M. Starr ${ }^{2 \dagger}$ and Sharon Abrahams ${ }^{1,3}$

${ }^{1}$ Human Cognitive Neuroscience, School of Philosophy, Psychology and Language Science, University of Edinburgh, Edinburgh, UK

${ }^{2}$ Alzheimer Scotland Dementia Research Centre, University of Edinburgh, Edinburgh, UK

${ }^{3}$ Euan MacDonald Centre for Motor Neurone Disease Research, University of Edinburgh,

Edinburgh, UK

${ }^{4}$ Centre for Cognitive Ageing and Cognitive Epidemiology, University of Edinburgh, Edinburgh, UK

${ }^{5}$ Faculty of Medicine and Health Sciences, University of East Anglia, Norwich, UK tDeceased

Word Count: 3661

* Correspondence to: R. Radakovic, University of East Anglia, Queens Building, Norwich, UK, NR4 7TJ

Email addresses: r.radakovic@uea.ac.uk, radakovic.ratko@gmail.com Tel: +441603591441 


\section{Abstract}

Objective: Apathy is a prominent syndrome across neurodegenerative diseases. The Dimensional Apathy Scale (DAS) assesses three apathy subtypes, Executive, Emotional, Initiation and is sensitive and valid in amyotrophic lateral sclerosis (ALS), Alzheimer's Disease (AD) and Parkinson's Disease. This study describes the development of the brief DAS (b-DAS), which will enable apathy to be swiftly detected in the clinic. Method: 102 ALS and 102 AD patients' previously collected data was used. Mokken analyses was performed on item-level data of each informant/carer-rated DAS subscale (Executive, Emotional and Initiation) for the initial scale reduction. Item-total correlational analyses against standard apathy (convergent validity criteria) and depression (divergent validity criteria) measures and qualitative examination of items aided final item selection. Receiver Operating Curve Analysis determined optimal cut-offs for the reduced subscales. Results: Mokken analyses suggested unidimensionality of each DAS subscale. Three items were removed that failed to satisfy monotone homogeneity model requirements, 3 items were removed due to validity criteria not being met and 6 items were removed due to a combination of lower item scalability and item-total correlations. Item-theme examination further reduced the b-DAS to 9 items, 3 per subscale, with a supplemental awareness deficit assessment being added. Sensitivity and specificity-based optimal cut-offs were calculated for each b-DAS subscale. Conclusions: The study presents the b-DAS, an informant/carerbased robust yet short multidimensional apathy instrument with good convergent and divergent validity, with recommended clinical cut-offs. The b-DAS is appropriate for use in the clinic and for research to quickly and comprehensively screen for apathy subtype impairments.

Keywords: apathy; assessment; short form; dementia; amyotrophic lateral sclerosis 


\section{Introduction}

Apathy is defined as a lack of motivation toward goal directed behavior (Marin, 1991) and has been shown to be prevalent in neurodegenerative and neuropsychiatric disease, such as dementia, amyotrophic lateral sclerosis (ALS), Parkinson's Disease, traumatic brain injury and schizophrenia (Chase, 2011; Radakovic \& Abrahams, 2018; Selbæk, Engedal \& Bergh, 2013; Strong et al., 2017). It can often manifest in cognitive, behavioural and emotional domains, for example through withdrawal, inertia, lack of initiative or avolition, blunting or reduction of emotions or asociality. Apathy has been shown to be associated with impaired activities of daily living, carer distress or burden, quality of life and survival in neurodegenerative disease (e.g. Starkstein et al., 2006; Kamiya et al., 2014; Watermeyer et al., 2015; Caga et al, 2016).

It is now established that apathy is a multidimensional syndrome, composed of different subtypes of demotivation. These subtypes are thought to have distinct neural substrates and several models relating to neural circuitry have been proposed, particularly in relation to the prefrontal cortex-basal ganglia networks (e.g. Levy \& Dubois, 2006; Levy, 2012). Apathy as a syndrome can be practically surmised through a Dimensional Apathy Framework (Radakovic \& Abrahams, 2018), which is comprised of a triadic structure including Executive apathy, a lack of motivation for planning, attention or organisation, Emotional apathy, a lack of emotional motivation (indifference or emotional neutrality) and Initiation apathy, a lack of motivation for self-generation of thoughts or actions. These apathy subtypes are assessed using the Dimensional Apathy Scale (DAS; Radakovic, \& Abrahams, 2014), which is a multidimensional apathy instrument that quantifies apathy subtypes independent of physical disability and so is particularly suitable for patients with motor disorders (Radakovic et al., 2016; Santangelo et al., 2017a; Santangelo et al., 2017b; Radakovic, Davenport, Starr 
\& Abrahams, 2018). It has been shown to differentiate apathy subtype profiles in different neurodegenerative diseases, with only Initiation apathy being characteristic of amyotrophic lateral sclerosis (ALS; Radakovic et al., 2016) and a combination of Executive and Initiation apathy being characteristic of Parkinson's disease (Radakovic et al., 2018) both independent of physical disability. In terms of cognitive dysfunction, Initiation and Emotional apathy have been shown to associate with executive dysfunction and emotional recognition deficits in ALS (Radakovic et al., 2017b). In Parkinson's disease, Executive apathy has been shown to associate with activities of daily living and quality of life (Radakovic et al., 2018; D'Iorio et al., 2017). Further, research in Alzheimer's Disease (AD) seem to show a global apathy over all subtypes (Radakovic et al., 2017a). The Dimensional Apathy Framework recognizes insight or awareness as an integrated element to apathy presentation (Radakovic \& Abrahams, 2018). In accord with other studies (Starkstein, 2014) insight or awareness can be assessed using specific assessment or self-informant/carer discrepancy scores. Further, the global apathy subtype profile observed in AD was found to be typified by distinctive deficits in awareness of apathy subtypes more localised to Executive and Initiation apathy (Radakovic et al., 2017a), with other research finding distinct apathy associated awareness deficits in Frontotemporal dementia (Fernández-Matarrubia et al., 2018). Therefore, the application of subtype assessment and this framework has furthered our understanding and efficacy in assessment of syndromic apathy.

Clinicians are often constrained by time when administering assessments to patients and/or their carers, having to manage a variety of assessments to attain a more complete clinical picture. This calls for shorter yet comprehensive tools. With the importance of multidimensional apathy assessment being repeatedly reaffirmed (Levy, 2012; Costa et al., 2017; Lanctôt et al., 2017), it would therefore be advantageous both in research and clinically 
to produce a brief version of the DAS. Item response theory (IRT) can be used to gain understanding of the properties of the individual items, how they function relative to one another and particularly whether the items form a hierarchy within each subscale factor (Mooij, 2012). One particular form of non-parametric IRT called Mokken scaling can be used in scale development as a tool for the identification of unidimensional sets of items and to obtain additional information about the relationship between test items within such scales. Mokken scaling can be applied in scale design or in the development of multi-item questionnaires measuring health constructs (Sijtsma et al., 2008), for example scale reduction of the General Health Questionnaire (Watson et al. 2008) and Addenbrooke's Cognitive Examination-III (Hsieh et al. 2015).

The aim of this study was to apply data driven scaling methods, to derive a 9 item short form of the DAS, the brief DAS (b-DAS), suitable for use within research settings and the clinic. Previous research employing Mokken analysis has reduced scales or assessments by over 50\% (e.g. Watson et al. 2008, Hsieh et al. 2015). To ensure that each subscale was equally represented and that the breath of characteristics was captured for each apathy subtype, 9 items (3 items per subscale) was chosen as the number of items for the b-DAS. Data was extracted from studies of ALS and AD to ensure that the new scale is appropriate for use in both patients with a selective and more global apathy profiles. Data was compared to a standard apathy measure and a depression scale to determine convergent and divergent validity respectively.

\section{Methods}

\section{Participants and procedure}


Previously collected carer data from 102 ALS patients (Radakovic et al., 2016; Radakovic et al., 2017b) and 102 AD patients (Radakovic et al., 2017a) was collated giving a total sample size of 204 and have been previously described (see Radakovic et al., 2016, Radakovic et al., 2017a; Radakovic et al., 2017b).

All participants' carers completed the following assessments about the patients. The DAS (Radakovic \& Abrahams, 2014) is a 24 item multidimensional apathy measure composed of 3 subscales- Executive, Emotional and Initiation (available through http://das.psy.ed.ac.uk/). The DAS was created to better profile apathy subtypes in people with neurodegenerative and neurological disease. Items are scored on a 4 point Likert scale and the scale takes approximately 5 minutes to complete. The items are summed for each subscale, with the total subscale score ranging from 0 (least apathetic) to 24 (most apathetic), with a DAS total score ranging from 0 to 72 . Age and education specific cut-offs have been calculated for AD and ALS (Radakovic et al., 2016; Radakovic et al., 2017a). The Apathy Evaluation Scale (AES; Marin, Biedrzycki \& Firinciogullari, 1991) is an 18 item, onedimensional, gold standard apathy measure. Items are scored on a 4 point Likert scale with a total score ranging from 18 (least apathetic) to 72 (most apathetic), with cut-offs for abnormality available (Marin et al., 1991; Clarke et al., 2007). Geriatric Depression ScaleShort form (GDS-15; Brown \& Schinka, 2005), a 15 item screen used to assess patients' depression. Each item was dichotomously scored (Yes/No) with the total score ranged from 0 (least depressed) to 15 (most depressed). A cut-off of $>6$ was used as an indication of depression (Wancata et al., 2006).

\section{Statistical Analysis}

All statistical analysis was performed using R 3.3.2, unless otherwise stated. 


\section{Item response analysis}

Mokken scaling analysis (Watson et al. 2012) was used to examine the hierarchy of items on each subscale of the informant/carer-rated Dimensional Apathy Scale (DAS) and to help derive the b-DAS. This method provides an indication of item difficulty and discrimination. Item difficulty refers to the ease with which the item is correctly responded to or endorsed. Item mean scores are used to represent the level of item difficulty. Discrimination refers to how precisely an item can differentiate between respondents with low levels of latent trait and those with high levels of latent trait. Discrimination also indicates the degree of association between items and the scale construct. With regard to scale development Mokken scaling analysis can be used to identify and select items with good discrimination in specific populations. Ideally scales would consist of items of high discrimination with different levels of difficulty to ensure differences and changes along the spectrum of ability or latent trait will be detected (Mungas \& Reed, 2000).

The fit of the DAS items to Mokken scaling properties was assessed by examining whether they conformed to the three assumptions of the monotone homogeneity model (MHM); unidimensionality, local stochastic independence and monotonicity. These assumptions were examined using a hierarchical clustering algorithm, scalability coefficients and latent monotonicity in the Mokken package in R (van der Ark, 2007).

Automated item selection procedure (AISP). AISP is used to partition items into scales, or groups or related items measuring a common latent trait, using a hierarchical clustering algorithm. AISP is a bottom-up sequential item selection method based on the strength of association between the items and the latent trait. The selection procedure starts with the pair of items with the highest positive item-pair scalability coefficient $\left(H_{i j}\right)$ and continues until no 
further items meet this criterion. Any items remaining can be selected to form a new scale in the same way. Items remaining out with a scale are considered unscalable (Sijtsma \& Molenaar, 2002).

Scalability coefficients. Scalability coefficients for items, item-pairs and the scale as a whole are calculated and used as criteria for partitioning items into scales and as measures of strength of the scales. The item scalability coefficient $\left(H_{i}\right)$ reflects item discrimination and is equivalent to item-test correlation or a factor loading. Item-pair scalability coefficients $\left(H_{i j}\right)$ expresses the joint scalability of item pairs and are applied in the AISP as described above. The strength of the overall scale is reflected by the scalability coefficient $(H)$. A general rule of thumb for interpretation of $\mathrm{H}$ has been described: scales with $H<.30$ are not considered unidimensional, $.30 \geq H<.40$ 'weak', $.40 \geq H<.50$ 'moderate', and $H>.50$ 'strong' (Mokken, 1971, Meijer \& Baneke, 2004).

Latent monotonicity. Monotonicity allows respondents to be ordered on the latent trait with respect to the summed score on the scale. Items violating this assumption can be identified and removed if necessary.

\section{Item-level psychometric analysis}

Item-total correlational analysis was used to examine convergent and divergent validity criteria of the DAS items and further stratify item selection for the brief DAS.

An acceptable convergent validity criterion was determined through DAS items correlations with the AES total score of $r \geq .5$. An acceptable divergent validity criterion was determined through the DAS item correlations with GDS total score of $\mathrm{r}<.5$. 
Receiver Operating Characterise (ROC) Curve Analysis

Receiver operating characteristic (ROC) curve analysis was performed using MedCalc for Windows, version 18.0 (MedCalc Software, Ostend, Belgium). Diagnosis of apathy was two tiered, where patients had to be classified as apathetic on both the full informant/carer-rated DAS subscales and the gold standard informant/carer AES only. Those that were impaired independently on each scale were not classified as apathetic. On this basis sensitivity, specificity, positive predictive value (PPV), negative predictive value (NPV) and positive likelihood ratios (+LR) for different criterion (cut-offs) were calculated for each b-DAS subscale.

\section{Results}

\section{Demographics}

Patient group clinical and demographic descriptors can be found in Table 1.

Table 1. Demographic, apathy and depression descriptors for AD and ALS patients

\begin{tabular}{lllll}
\hline & ALS $(n=102)$ & AD $(n=102)$ & $p$ Value & Total $(n=204)$ \\
\hline Age (Mean, SD) & $63.8(11.0)$ & $78.2(8.5)$ & $<.001$ & $71.0(12.1)$ \\
Gender (Male/Female) & $71 / 31$ & $52 / 50$ & $<.01$ & $123 / 81$ \\
Years of Education (Mean, SD) & $13.2(2.1)$ & $13.2(3.7)$ & $\mathrm{NS}$ & $13.2(3.3)$ \\
Age Onset years (Mean, SD) & $59.4(11.5)$ & $74.6(8.2)$ & $<.001$ & $67.6(12.4)$ \\
Disease Duration months (median, & $48(72)$ & $36(48)$ & $\mathrm{NS}$ & $48(60)$ \\
IQR) & & & & \\
AES Total (Mean, SD) & $33.2(10.8)$ & $51.7(11.5)$ & $<.001$ & $42.4(14.4)$ \\
GDS-15 Total (Mean, SD) & $6.8(4.7)$ & $7.3(4.4)$ & $\mathrm{NS}$ & $7.1(4.5)$ \\
\hline
\end{tabular}

$\mathrm{SD}=$ Standard Deviation; IQR = Interquartile Range; AES = Apathy Evaluation Scale; GDS-15 = Geriatric Depression Scale-Short form; NS = Not Significant 
The most common informant/carer relationship for both patient groups was a spouse or partner. AD and ALS patients were matched for years of education, but significantly differed in age, gender distribution and age of onset (see Table 1). Further, AD patients were significantly more apathetic than ALS patients on the AES, where $75.5 \%$ of AD patient compared to $25.5 \%$ of ALS patients displayed apathy (based on the AES cut-offs). No such difference found in relation to depression, where $54.9 \%$ of AD patients and $48.0 \%$ of ALS patients displayed depression (based on the GDS-15 cut-offs). Correlational analysis found no significant relationship between the AES or GDS-15 and age or age onset, and no gender differences were observed in terms of the AES and GDS-15. 


\section{Item Response Analysis, Correlational Analysis and Final Item selection}

Table 2. Executive, Emotional and Initiation subscale items ordered by discrimination $\left(H_{i}\right)$. Item difficulty and correlation with AES and GDS-15 in full sample ( $n=204)$

\begin{tabular}{|c|c|c|c|c|c|}
\hline \multicolumn{6}{|c|}{ Executive } \\
\hline Item & Label & $H_{i}$ & Difficulty & AES & GDS-15 \\
\hline no. & & & & Correlation & Correlation \\
\hline 21 & S/he finds it difficult to keep his/her mind on things & 0.760 & 1.485 & 0.800 & 0.371 \\
\hline 17 & $\begin{array}{l}\text { When doing a demanding task, s/he has difficulty working } \\
\text { out what s/he has to do }\end{array}$ & 0.718 & 1.377 & 0.771 & 0.295 \\
\hline 19 & $\begin{array}{l}\text { S/he gets easily confused when doing several things at } \\
\text { once }\end{array}$ & 0.708 & 1.569 & 0.734 & 0.278 \\
\hline 10 & S/he is able to focus on a task until it is finished & 0.699 & 1.436 & 0.795 & 0.380 \\
\hline 1 & S/he needs a bit of encouragement to get things started & 0.699 & 1.436 & 0.764 & 0.394 \\
\hline 11 & $\mathrm{~S} /$ he lacks motivation & 0.660 & 1.559 & 0.753 & 0.523 \\
\hline 23 & $\mathrm{~S} / \mathrm{he}$ is easily distracted & 0.649 & 1.544 & 0.625 & 0.338 \\
\hline 6 & S/he stares in to space & 0.630 & 1.353 & 0.642 & 0.437 \\
\hline \multicolumn{6}{|c|}{ Emotional } \\
\hline Item & Label & $\overline{H_{i}}$ & Difficulty & AES & GDS-15 \\
\hline no. & & & & Correlation & Correlation \\
\hline 5 & S/he is concerned about how his/her family feel & 0.428 & 1.461 & 0.552 & 0.120 \\
\hline 7 & $\begin{array}{l}\text { Before s/he does something s/he thinks about how others } \\
\text { would feel about it }\end{array}$ & 0.417 & 2.044 & 0.552 & 0.242 \\
\hline 24 & S/he is indifferent to what is going on around him/her & 0.402 & 1.103 & 0.699 & 0.355 \\
\hline 12 & S/he struggles to empathise with other people & 0.381 & 1.162 & 0.579 & 0.335 \\
\hline 9 & When $\mathrm{s} /$ he receives bad news $\mathrm{s} / \mathrm{he}$ feels bad about it & 0.334 & 1.098 & 0.412 & 0.048 \\
\hline $3 *$ & $\mathrm{~S} /$ he expresses his/her emotions & 0.268 & 1.667 & 0.335 & 0.132 \\
\hline $20^{*}$ & $\begin{array}{l}\text { S/he becomes emotional easily when watching something } \\
\text { happy or sad on TV }\end{array}$ & 0.213 & 1.956 & 0.180 & -0.109 \\
\hline $15 \ddagger$ & $\begin{array}{l}\mathrm{S} / \text { he is unconcerned about how others feel about his/her } \\
\text { behaviour }\end{array}$ & 0.173 & 1.221 & 0.276 & 0.175 \\
\hline \multicolumn{6}{|c|}{ Initiation } \\
\hline Item & Label & $H_{i}$ & Difficulty & AES & GDS-15 \\
\hline no. & & & & Correlation & Correlation \\
\hline 4 & S/he thinks of new things to do during the day & 0.682 & 2.373 & 0.723 & 0.430 \\
\hline 13 & S/he sets goals for him/herself & 0.665 & 2.157 & 0.745 & 0.430 \\
\hline 16 & S/he acts on things s/he has thought about during the day & 0.627 & 1.804 & 0.691 & 0.337 \\
\hline 14 & S/he tries new things & 0.601 & 2.279 & 0.671 & 0.420 \\
\hline 2 & S/he contacts his/her friends & 0.593 & 1.995 & 0.732 & 0.374 \\
\hline 8 & S/he plans his/her days activities in advance & 0.590 & 2.221 & 0.583 & 0.346 \\
\hline 18 & S/he keeps him/herself busy & 0.590 & 1.593 & 0.803 & 0.524 \\
\hline 22 & $\mathrm{~S} / \mathrm{he}$ is spontaneous & 0.501 & 2.034 & 0.503 & 0.362 \\
\hline
\end{tabular}


Executive. The AISP partitioned all 8 items of the Executive subscale into one scale. The scalability coefficients of the items were examined. All item-pair scalability coefficients $\left(H_{i j}\right)$ were non-negative and all item scalability coefficients were above 0.3 . There were no significant violations of monotonicity. The items of the Executive subscale form a Mokken scale meeting MHM criteria $(H=0.680, S E=0.026)$. See Table 2 for Executive items ordered by $H_{i}$.

Correlational analysis in Table 2 showed that only item 11 did not meet the divergent validity criterion and was subsequently removed. A further examination of item scalability in combination with correlational analysis resulted in removal of items 6,23 , and 1 .

Examination of the remaining items revealed that item 19 held similarities in terms of confusion over management of tasks with item 17. Therefore, item 19 was removed and item 10 utilised in its place as it examined more direct motivational elements to do with planning and focus to finish a tasks. This resulted in retention of items 21,17 and 10.

Emotional. The AISP partitioned five items into one scale (items 5, 7, 9, 12 and 24), two items into a separate scale (items 3 and 20) and determined the remaining one item (item 15) to be unscalable. The scalability coefficients of the five items of scale 1 were examined. These 5 items met the criteria for inclusion in a unidimensional Mokken MHM scale $(\mathrm{H}=0.501, S E=0.038)$. See Table 2 for Emotional items ordered by $H_{i}$.

Correlational analysis in Table 2 showed that out of these items only item 9 did not meet the convergence validity criterion, and was subsequently removed. 
Examination of the remaining items revealed that item 12 was similar to both items 5 and 7 , in terms of empathy towards family or others. Therefore, item 12 was removed and item 24 was utilised in its place, as it explicitly examined indifference, an important facet of Emotional apathy. This resulted in retention of items 5, 7 and 24.

Initiation. All items were partitioned into one scale through the AISP. Scalability coefficients all met the required criterion for inclusion in a Mokken scale with non-negative item-pair scalability coefficients, item scalability coefficients greater than .30 for all items and a scale $H$ of 0.615 ( $S E=0.031)$. There were no violations of monotonicity. See Table 2 for Initiation items ordered by $H_{i}$.

Correlational analysis in Table 2 showed that only item 18 did not meet the divergent validity criterion and was subsequently removed. A further examination of item scalability in combination with correlational analysis resulted in removal of items 22,8 and 2.

Examination of the remaining items revealed that item 14 was similar to item 4 , in relation to motivation for new things. Therefore, item 14 was removed and item 16 was utilised in its place, as it assesses the ability to act on things they have already thought about. This resulted in retention of items 4,13 and 16.

This resulted in the b-DAS composed of 9 items, equally distributed over Executive, Emotional and Initiation apathy subscales. For each item a supplementary awareness deficit question was added (“Are they aware of this specific difficulty?”), which is answered dichotomously (Yes/No) when the item is scored on the upper two points of the Likert Scale. 
Summation of the awareness deficit questions responses ("No") for each apathy subtype can be $0=$ Intact Awareness, $1=$ Mild Awareness Deficit, $2=$ Moderate Awareness Deficit and 3 $=$ Severe Awareness Deficit.

\section{ROC Curve analysis of b-DAS}

Table 3. Sensitivity, Specificity, positive and negative predictive values and positive likelihood ration for b-DAS Subscale cut-offs $(\mathrm{N}=\mathbf{2 0 4})$

\begin{tabular}{|c|c|c|c|c|c|}
\hline $\begin{array}{l}\text { b-DAS } \\
\text { Subscale }\end{array}$ & $\begin{array}{l}\text { Criterion } \\
\text { (Cut-off) }\end{array}$ & Sensitivity & Specificity & PPV (NPV) & $+\mathrm{LR}$ \\
\hline \multirow[t]{4}{*}{ Executive } & 3 & 100.0 & 74.6 & $72.6(100.0)$ & 3.94 \\
\hline & 4 & 98.8 & 86.9 & $83.5(99.1)$ & 7.53 \\
\hline & 5 & 93.9 & 97.5 & $96.2(96.0)$ & 38.19 \\
\hline & 6 & 78.1 & 99.2 & $98.5(87.1)$ & 95.22 \\
\hline \multirow[t]{4}{*}{ Emotional } & 4 & 98.2 & 66.7 & $51.5(99.0)$ & 2.94 \\
\hline & 5 & 94.4 & 77.3 & $60.0(97.5)$ & 4.17 \\
\hline & 6 & 87.0 & 94.0 & $83.9(95.3)$ & 14.51 \\
\hline & 7 & 55.6 & 98.0 & $90.9(86.0)$ & 27.78 \\
\hline \multirow[t]{4}{*}{ Initiation } & 5 & 100.0 & 68.5 & $73.8(100.0)$ & 3.18 \\
\hline & 6 & 96.9 & 84.3 & $84.5(96.8)$ & 6.15 \\
\hline & 7 & 82.3 & 92.6 & $90.8(85.5)$ & 11.11 \\
\hline & 8 & 55.2 & 97.2 & $94.6(70.9)$ & 19.87 \\
\hline
\end{tabular}

PPV $=$ Positive Predictive Value; NPV = Negative Predictive Value + LK $=$ Positive Likelihood Ratio. Criteria in bold were considered optimal either in terms of sensitivity or specificity.

Table 3 shows results of sensitivity, specificity, PPV, NPV and +LR for each cut-off for each b-DAS subscale. For the Executive apathy a cut-off of 4 was found to be optimal for sensitivity and a cut-off 5 was optimal for specificity, both with high predictive values (PPV and NPV). Therefore the recommended cut-off for this b-DAS subscale was determined as $\geq$ 4. For Emotional apathy a cut-off of 5 was found to be optimal for sensitivity and a cut-off 6 
was optimal for specificity, with the latter having a lower PPV than the former, but comparable NPV. The recommended cut-off for this b-DAS subscale was determined as $\geq 5$. Finally, for Initiation apathy a cut-off of 6 was found to be optimal for sensitivity and a cutoff 7 was optimal for specificity, with comparable PPV and NPV. The recommended cut-off for this b-DAS subscale was determined as $\geq 6$. The selected cut-offs have high sensitivity and specificity with likelihood ratios indicating that scores on or above these cut-offs are more than 4 times likely to be from a patient with apathy than without.

\section{Discussion}

We present the b-DAS, an informant/carer-based, 9-item multidimensional apathy instrument to measure Executive, Emotional and Initiation apathy subtypes, in addition to insight/awareness deficit, of people living with neurological disease. The inclusion of the Awareness (or Insight) deficit measure ensures that self-knowledge relating motivational problems can be quantified for each subtype, which may have particular relevance for clinical intervention and management of this symptom. Each subscale has a minimum score of 0 (least apathy) maximum score of 9 (most apathy), with clinical cut-offs for Executive $\geq 4$, Emotional $\geq 5$ and Initiation $\geq 6$ apathy subscales, taking in to account sensitivity and specificity as well as NPV and PPV. This is further supplemented by Awareness deficit classification for each subtype ranging from 0 as "Intact" to 3 as "Severe". This reflects the importance of awareness of demotivation in diseases such as AD or other dementias (Radakovic \& Abrahams, 2018). In comparison with the original DAS, the b-DAS takes less than 5 minutes to complete, increasing the efficiency of assessment (the DAS and b-DAS are available through http://das.psy.ed.ac.uk/ or by contacting the lead author). 
The Mokken Scaling IRT analysis of the DAS showed that most item scalability coefficients were above the recommended threshold level for inclusion in a Mokken scale for creation of the b-DAS, indicating adequate sample size (Straat et al. 2014). There was an exception in relation to the Emotional apathy subscale, where some items were below the scalability coefficient threshold of 0.3 and were partitioned in to different scales. Previous research using the DAS has suggested that the Emotional apathy may be composed of subdomains of "Individual" Emotional apathy relating to personal and internal emotional motivation (or "inertia") and "Social" Emotional apathy relating to motivation towards external and complex interactions (M'Barek et al., In Press). The b-DAS includes items from both these possible Emotional apathy subdomains, however research is needed to explore their reliability and consistency. Further, inclusion of both the AD and ALS patient groups gave a large enough sample size $(n=204)$ to allow for a robust Mokken scaling analysis to be performed. A simulation study investigating adequate sample sizes for Mokken scaling determined that the strength of item scalability coefficients, $H_{i}$, is inversely proportional to sample size which serves as a good indicator of adequate sample size (Straat, 2012). Further, while there are other parametric IRT methods available (e.g. Rasch analysis and the twoparameter model extension), our use of the non-parametric Mokken analysis was driven by the less stringent assumptions of this method in relation to the item responses (Van Schuur, 2003). This was considered favourable when working with a patient population as responses on items are inherently heterogeneous. Additionally, as the profiles of apathy are different in ALS and AD, this covers the breadth of demotivational characteristics within disease.

Further, the inclusion of convergence and divergence criteria against gold-standard apathy and depression measures ensured that items selected for the b-DAS were of a high validity. While there is a degree of overlap between one-dimensional apathy and depression (Landes 
et al., 2001; Tagariello et al., 2009), an emerging consensus is that in terms of subtypes, Executive and Initiation apathy may be associated to a degree with depression, whereas Emotional apathy diverges in terms of emotional neutrality (Radakovic et al., 2016; Radakovic et al., 2017a; Radakovic et al., 2018). This points towards the importance and necessity of further research to specifically elucidate the relationship of apathy subtypes and depression.

The b-DAS subscale cut-offs that were selected balanced highest sensitivity and specificity, to maximise identification of apathy subtypes. The cut-offs that are recommended marginally favoured sensitivity over specificity, as overlooking apathetic deficits may have more negative effects than false detection, in terms for example activities of daily living, carer distress or burden, quality of life and survival (e.g. Starkstein et al., 2006; Kamiya et al., 2014; Watermeyer et al., 2015; Caga et al, 2016).

Furthermore, an awareness deficit assessment has been added to the b-DAS, which was driven by its importance as emerging concept of demotivation-based self-awareness (Radakovic \& Abrahams, 2018). The apathy subtype profile in AD seems to be characterised by this awareness deficit (Radakovic et al., 2017a), when compared to ALS and Parkinson's disease (Radakovic et al., 2016; Santangelo et al., 2017a; Santangelo et al., 2017b; Radakovic et al., 2018). The awareness deficit assessment within the b-DAS predominantly examines self-knowledge of specific impairments associated with apathy (Marková \& Berrios, 2011). However, there are also elements of the wider concept of insight that are assessed (Marková $\&$ Berrios, 2011), in that there is a quantitative and qualitative appraisal by the informant/carer of the patient's awareness of this specific "difficulty" (i.e. highly endorsed bDAS item). Additionally, Starkstein (2014) found that anosognosia (or loss of 
insight/awareness) is associated with apathy and might be implicit in lower adherence to pharmacological and non-pharmacological treatment. Assessment of the insight/awareness deficit in the b-DAS therefore increases the practical applicability of this measure in clinic, but would need to be further explored to determine specific the distribution of different apathy awareness deficits as well as clinical cut-offs.

In conclusion, the b-DAS is a comprehensively designed, short, informant/carer-rated tool that assesses different subtypes of apathy and associated awareness deficits that can be used quickly in clinic and research. We present recommended cut-offs for each b-DAS subscale that can be applied to neurodegenerative, as well as other neurological, conditions. Future clinical practice and research should utilise the b-DAS to explore its clinical applicability, so as to determine how demotivation impacts clinical decision making, treatment choice or adherence, quality of life of patients and carer burden. This will help guide and design interventions or strategies to manage syndromic apathy.

\section{Acknowledgments}

The authors would like to thank all the participants and their families. This work was supported by the University of Edinburgh, Anne Rowling Regenerative Neurology Clinic, Euan MacDonald Centre for Motor Neurone Disease Research and Alzheimer Scotland Dementia Research Centre, as well as the Motor Neurone Disease Scotland and the Motor Neurone Disease Association.

\section{Declaration of interest}

The authors have no conflict of interest to report. 


\section{References}

Brown, L. M., \& Schinka, J. A. (2005). Development and initial validation of a 15 - item informant version of the Geriatric Depression Scale. International Journal of Geriatric Psychiatry, 20(10), 911-918.

Caga, J., Turner, M. R., Hsieh, S., Ahmed, R. M., Devenney, E., Ramsey, E., ... \& Kiernan, M. C. (2016). Apathy is associated with poor prognosis in amyotrophic lateral sclerosis. European journal of neurology, 23(5), 891-897.

Chase, T. N. (2011). Apathy in neuropsychiatric disease: diagnosis, pathophysiology, and treatment. Neurotoxicity research, 19(2), 266-278.

Clarke, D. E., Reekum, R. V., Simard, M., Streiner, D. L., Freedman, M., \& Conn, D. (2007). Apathy in dementia: an examination of the psychometric properties of the apathy evaluation scale. The Journal of neuropsychiatry and clinical neurosciences, 19(1), 57-64.

Costa, A., Bak, T., Caffarra, P., Caltagirone, C., Ceccaldi, M., Collette, F., ... \& Duzel, E. (2017). The need for harmonisation and innovation of neuropsychological assessment in neurodegenerative dementias in Europe: consensus document of the Joint Program for Neurodegenerative Diseases Working Group. Alzheimer's research \& therapy, 9(1), 27. D'Iorio, A., Vitale, C., Piscopo, F., Baiano, C., Falanga, A. P., Longo, K., ... \& Santangelo, G. (2017). Impact of anxiety, apathy and reduced functional autonomy on perceived quality of life in Parkinson's disease. Parkinsonism \& related disorders, 43, 114-117.

Fernández-Matarrubia, M., Matías-Guiu, J. A., Cabrera-Martín, M. N., Moreno-Ramos, T., Valles-Salgado, M., Carreras, J. L., \& Matías-Guiu, J. (2018). Different apathy clinical profile and neural correlates in behavioral variant frontotemporal dementia and Alzheimer's disease. International journal of geriatric psychiatry, 33(1), 141-150. 
Hsieh, H., McGrory, S., Leslie, F., Dawson, K., Ahmed, S., Butler, C. R., ... \& Hodges, J. R. (2015). The Mini-Addenbrooke's Cognitive Examination: A New Assessment Tool for Dementia. Dementia and Geriatric Cognitive Disorders, 39(1-2), 1-11.

Kamiya, M., Sakurai, T., Ogama, N., Maki, Y., \& Toba, K. (2014). Factors associated with increased caregivers' burden in several cognitive stages of Alzheimer's disease. Geriatrics \& gerontology international, 14(S2), 45-55.

Lanctôt, K. L., Agüera-Ortiz, L., Brodaty, H., Francis, P. T., Geda, Y. E., Ismail, Z., ... \& Politis, A. M. (2017). Apathy associated with neurocognitive disorders: recent progress and future directions. Alzheimer's \& dementia: the journal of the Alzheimer's Association, 13(1), 84-100.

Landes, A. M., Sperry, S. D., Strauss, M. E., \& Geldmacher, D. S. (2001). Apathy in Alzheimer's disease. Journal of the American Geriatrics Society, 49(12), 1700-1707. Levy, R. (2012). Apathy: a pathology of goal-directed behaviour. A new concept of the clinic and pathophysiology of apathy. Revue neurologique, 168(8-9), 585-597.

Levy, R., \& Dubois, B. (2006). Apathy and the functional anatomy of the prefrontal cortexbasal ganglia circuits. Cerebral cortex, 16(7), 916-928.

M'Barek, L., Radakovic, R., Noquet, M., Laurent, A., \& Allain, P. (In Press). Different aspects of emotional processes in apathy: Application of the French translated dimensional apathy scale. Current Psychology. doi: 10.1007/s12144-017-9775-5

Marková, I. S., \& Berrios, G. E. (2011). Awareness and insight in psychopathology: An essential distinction? Theory \& Psychology, 21(4), 421-437.

Marin, R. S. (1991). Apathy: a neuropsychiatric syndrome. The Journal of Neuropsychiatry and Clinical Neuroscience, 3, 243-254.

Marin, R. S. Biedrzycki, R. C., \& Firinciogullari, S. (1991). Reliability and validity of the Apathy Evaluation Scale. Psychiatry Research, 38(2), 143-162. 
Meijer, R. R., \& Baneke, J. J. (2004). Analyzing psychopathology items: a case for nonparametric item response theory modeling. Psychological methods, 9(3), 354. Mokken, R. J. (1971). A theory and procedure of scale analysis: With applications in political research (Vol. 1). Walter de Gruyter.

Mooij, T. (2012). A Mokken scale to assess secondary pupils' experience of violence in terms of severity. Journal of Psychoeducational Assessment, 30(5), 496-508.

Mungas, D., \& Reed, B. R. (2000). Application of item response theory for development of a global functioning measure of dementia with linear measurement properties. Statistics in Medicine, 19(11-12), 1631-1644

Radakovic, R., \& Abrahams, S. (2014). Developing a new apathy measurement scale: Dimensional Apathy Scale. Psychiatry research, 219(3), 658-663.

Radakovic, R., \& Abrahams, S. (2018). Multidimensional apathy: evidence from neurodegenerative disease. Current Opinion in Behavioral Sciences, 22, 42-49. Radakovic, R., Davenport, R., Starr, J. M., \& Abrahams, S. (2018). Apathy dimensions in Parkinson's disease. International journal of geriatric psychiatry, 33(1), 151-158.

Radakovic, R., Starr, J. M., \& Abrahams, S. (2017a). A novel assessment and profiling of multidimensional apathy in Alzheimer's disease. Journal of Alzheimer's Disease, 60(1), 5767.

Radakovic, R., Stephenson, L., Colville, S., Swingler, R., Chandran, S., \& Abrahams, S. (2016). Multidimensional apathy in ALS: validation of the Dimensional Apathy Scale. Journal of Neurology, Neurosurgery \& Psychiatry, 87(6), 663-669.

Radakovic, R., Stephenson, L., Newton, J., Crockford, C., Swingler, R., Chandran, S., \& Abrahams, S. (2017b). Multidimensional apathy and executive dysfunction in amyotrophic lateral sclerosis. Cortex, 94, 142-151. 
Santangelo, G., D’Iorio, A., Piscopo, F., Cuoco, S., Longo, K., Amboni, M., ... \& Vitale, C. (2017a). Assessment of apathy minimising the effect of motor dysfunctions in Parkinson's disease: a validation study of the dimensional apathy scale. Quality of Life Research, 26(9), $2533-2540$.

Santangelo, G., Siciliano, M., Trojano, L., Femiano, C., Monsurrò, M. R., Tedeschi, G., \& Trojsi, F. (2017b). Apathy in amyotrophic lateral sclerosis: insights from Dimensional Apathy Scale. Amyotrophic Lateral Sclerosis and Frontotemporal Degeneration, 18(5-6), 434-442.

Selbæk, G., Engedal, K., \& Bergh, S. (2013). The prevalence and course of neuropsychiatric symptoms in nursing home patients with dementia: a systematic review. Journal of the American Medical Directors Association, 14(3), 161-169.

Sijtsma, K., \& Molenaar, I. W. (2002). Introduction to nonparametric item response theory (Vol. 5). Sage.

Sijtsma, K., Emons, W. H., Bouwmeester, S., Nyklíček, I., \& Roorda, L. D. (2008).

Nonparametric IRT analysis of quality-of-life scales and its application to the world health organization quality-of-life scale (WHOQOL-Bref). Quality of Life Research, 17(2), 275290.

Starkstein, S. E. (2014). Anosognosia in Alzheimer's disease: diagnosis, frequency, mechanism and clinical correlates. Cortex, 61, 64-73.

Starkstein, S. E., Jorge, R., Mizrahi, R., \& Robinson, R. G. (2006). A prospective longitudinal study of apathy in Alzheimer's disease. Journal of Neurology, Neurosurgery \& Psychiatry, 77(1), 8-11.

Straat, J. H., van der Ark, L. A., \& Sijtsma, K. (2014). Minimum Sample Size Requirements for Mokken Scale Analysis. Educational and Psychological Measurement, 74(5), 809-822 
Straat, JH. (2012). Using Scalability Coefficients and Conditional Association to Assess Monotone Homogeneity. Ridderkerk: Ridderprint.

Strong, M. J., Abrahams, S., Goldstein, L. H., Woolley, S., Mclaughlin, P., Snowden, J., ... \& Rosenfeld, J. (2017). Amyotrophic lateral sclerosis-frontotemporal spectrum disorder (ALSFTSD): Revised diagnostic criteria. Amyotrophic Lateral Sclerosis and Frontotemporal Degeneration, 18(3-4), 153-174.

Tagariello, P., Girardi, P., \& Amore, M. (2009). Depression and apathy in dementia: same syndrome or different constructs? A critical review. Archives of Gerontology and Geriatrics, 49(2), 246-249.

Van der Ark, L. A. (2007). Mokken scale analysis in R. Journal of Statistical Software, 20 (11), 1-19.

Van Schuur, W. H. (2003). Mokken scale analysis: Between the Guttman scale and parametric item response theory. Political Analysis, 11(2), 139-163.

Wancata, J., Alexandrowicz, R., Marquart, B., Weiss, M., \& Friedrich, F. (2006). The criterion validity of the Geriatric Depression Scale: a systematic review. Acta Psychiatrica Scandinavica, 114(6), 398-410.

Watermeyer, T. J., Brown, R. G., Sidle, K. C., Oliver, D. J., Allen, C., Karlsson, J., ... \& Goldstein, L. H. (2015). Impact of disease, cognitive and behavioural factors on caregiver outcome in amyotrophic lateral sclerosis. Amyotrophic Lateral Sclerosis and Frontotemporal Degeneration, 16(5-6), 316-323.

Watson, R., Deary, I. J., \& Shipley, B. (2008). A hierarchy of distress: Mokken scaling of the GHQ-30. Psychological medicine, 38(4), 575-579.

Watson, R., van der Ark, L. A., Lin, L. C., Fieo, R., Deary, I. J., \& Meijer, R. R. (2012). Item response theory: how Mokken scaling can be used in clinical practice. Journal of Clinical Nursing, 21, 2736-2746. 